DOI: $10.17957 / \mathrm{IJAB} / 15.1790$

http://www.fspublishers.org

\title{
Optimization of Anti Phytophthora infestans Metabolites Produced by Myxococcus xanthus B25-I-1
}

\author{
Zhi Hua Wu ${ }^{1,2,3}$, Pu Yu Zhao ${ }^{1}$, Yi Xiu Ding ${ }^{1}$, Qiang Ma ${ }^{1}$, Xue Han Wang ${ }^{1}$ and Hui Rong Liu ${ }^{1 *}$ \\ ${ }^{1}$ College of Life Sciences, Inner Mongolia Agricultural University, Hohhot 010010, P. R. China \\ ${ }^{2}$ Baotou Teachers' College, Baotou 014030, P. R. China \\ ${ }^{3}$ Hospital of Baotou Medical College, Inner Mongolia University of Science and Technology, Baotou 014030, P. R. \\ China \\ "For correspondence: huirong_liu@imau.edu.cn; wuzhihuaqueen@163.com \\ Received 22 August 2020; Accepted 30 December 2020; Published 10 May 2021
}

\begin{abstract}
This study aimed to screen myxobacteria strains with strong activity against Phytophthora infestans and to study its secondary metabolites. Myxobacteria were isolated from a soil sample using the rabbit feces as bait. A strain inhibitive to $P$. infestans was identified by morphological observation and 16S rRNA sequence analysis. The fermentation parameters were optimized through a combination of univariate analysis and orthogonal optimization. The stability and antimicrobial activities of the secondary metabolites were determined using the filter paper method, and the effects on the development of the potato late blight and its pathogen were determined using the in vitro leaf method. The myxobacteria B25-I-1 isolated from a soil sample showed strong activity against $P$. infestans and was identified as Myxococcus xanthus. The optimal fermentation conditions of M. xanthus B25-I-1 were as follows: inoculum size of $10 \%$, shaking at $180 \mathrm{r} \mathrm{min}^{-1}$, incubation temperature of $32^{\circ} \mathrm{C}$, and incubation time of 8 days. The secondary metabolites were highly tolerant to high temperature, treatment with protease $\mathrm{K}$, and ultraviolet and natural lights. Besides $P$. infestans, the secondary metabolites also showed variant antagonistic effects against Escherichia coli, Staphylococcus aureus, Bacillus subtilis, Saccharomyces cerevisiae, Fusarium oxysporum, Rhizoctonia solani, and Sclerotinia sclerotiorum. The results of the potato late blight control on potato leaves indicated that the secondary metabolites could significantly inhibit the infection of detached potato leaves caused by $P$. infestans and caused no damage to the leaves of different varieties of potatoes by themselves. This study provides basic data for the the development of a biopesticide against potato late blight. (C) 2021 Friends Science Publishers
\end{abstract}

Keywords: Myxobacteria; Potato late blight; Myxococcus xanthus; Orthogonal optimization; Secondary metabolite

\section{Introduction}

Myxobacteria are gram-negative bacteria that undergo social behavior, complex multicellular behavior, and morphogenesis (Kim et al. 2016), and are higher prokaryotes (Sun et al. 2016), known for their predatory lifestyle (Livingstone et al. 2018). Myxobacteria are good resources for natural drug screening. With more than 100 core structures and over 500 derivatives been published to date (Herrmann et al. 2017). They have joined a large group of microbes with great potential for research and development (Diez et al. 2012). Moreover, the biosynthetic potential of myxobacteria is huge, and it is estimated that $50-100 \%$ of myxobacteria can synthesize biologically active secondary metabolites (Wenzel and Müller 2009). In prokaryotes ranked according to the number of bioactive secondary metabolites, myxobacteria follow only actinomyces and bacillus (Wang and Ma 2010), but the fraction of myxobacteria with bacteriostatic activity is higher than that of actinomycetes. Because myxobacteria can produce several bioactive secondary metabolites with novel structures and unique mechanism of action, they are considered the microfactories of secondary metabolites with biological activity. At present, myxobacteria have become extremely active subjects of domestic and international research (Weissman and Müller 2009).

Potato is the fourth largest food crop worldwide after rice, wheat and maize, and it is the fourth largest staple food in China. Potato late blight is the most serious disease of potato and has seriously hindered the production and industrialization of potato. At present, potato late blight is mainly controlled by chemical agents, and the commonly used agents include metalaxyl mancozeb, metalaxyl, and oxadixyl mancozeb (Wu et al. 2020). The extensive use of chemical pesticides damages the environment. Therefore, it is extremely urgent to develop environmentally friend pesticides with high efficiency to control potato late blight.

Potato late blight is caused by Phytophthora infestans

To cite this paper: Wu ZH, PY Zhao, YX Ding, Q Ma, XH Wang, HR Liu (2021). Optimization of Anti Phytophthora infestans metabolites produced by Myxococcus xanthus B25-I-1. Intl J Agric Biol 25:1281-1291 
(Fry 2008), which belongs to Chromista, Oomycota, Mastigomycotina, Ommycetes, Peronosporales, Pythiaceae. The host range of $P$. infestans is relatively narrow, and $P$. infestans mainly infecting potatoes, tomatoes and other 50 eggplant plants. On these hosts, $P$. infestans usually reproduce by asexual reproduction, and also by sexual oospores in the presence of two mating types, A1 and A2 (Wu 2018). P. infestans causes a devastating disease wherein the potato stems and leaves die and the tuber rots, mainly by invading leaves, stems, and potato stalks (Haas et al. 2009).

In the present study, a myxobacteria strain (laboratory number for the strain B25-I-1) with significant inhibitory effects on $P$. infestans was isolated from a soil sample collected from the Baotou area, Inner Mongolia Autonomous Region. It was identified by morphological observation and 16S rDNA sequence analysis. The antimicrobial activities of the strain were analyzed, and its fermentation conditions were optimized through orthogonal experiments. The stability and antimicrobial activities of the bioactive secondary metabolites in the concentrated fermentation broth and their anti-disease effects on potato late blight were studied using the in vitro leaf method.

\section{Materials and Methods}

\section{Soil sampling}

A soil sample was obtained from grassland in the Baotou area of Inner Mongolia Autonomous Region. It was located at $40^{\circ} 18^{\prime} 38^{\prime \prime}$ north latitude and $110^{\circ} 50^{\prime} 32^{\prime \prime}$ east longitude and was considered meadow salt soil. Soil was collected 1$10 \mathrm{~cm}$ below the surface using the five-point sampling method, and the sample was naturally air dried at room temperature.

\section{Bacterial and fungal strains}

Staphylococcus aureus CMCC26003, Bacillus subtilis 3-1, Escherichia coli DH-5 $\alpha$, Saccharomyces cerevisiae C2-2, P. infestans HQK8-3, Fusarium oxysporum, Rhizoctonia solani, Sclerotinia sclerotiorum and Verticillium dahliae were provided by our laboratory (Applied Environmental Microbiology Laboratory, Inner Mongolia Agricultural University).

\section{Induction, purification, and preservation of myxobacteria}

The soil samples that had been naturally air-dried and screened at 60 mesh were baked at $58^{\circ} \mathrm{C}$ for $1 \mathrm{~h}$ to remove the bacteria with poor heat resistance. Approximately $30 \mathrm{~g}$ of the soil sample was placed in a 90-mm Petri dish and soaked overnight at room temperature in a cycloheximide solution at a final concentration of $100 \mu \mathrm{g} \mathrm{mL} \mathrm{m}^{-1}$ to help remove mold and yeast. The pretreated soil samples were poured into a ST21CX solid culture dish and paved. Half of sterilized rabbit dung pellets were embedded in the soil sample, and the culture dishes were incubated at $30^{\circ} \mathrm{C}$ for 6 days. We observed the formation of myxobacteria fruiting bodies over this time (Liu et al. 2011).

Myxobacteria were identified and the borders of characteristic colonies or the heads of fruiting bodies were directly picked onto fresh VY/2 medium with a sterile inoculation needle to remove a portion of the larger and immobile bacteria and cultured at $30^{\circ} \mathrm{C}$ (Guo et al. 2007). This method required repeated transfer until there were no other bacteria growing in the medium.

The fruiting bodies of the strains were incubated in the CAS liquid medium and shaken for $36 \mathrm{~h}$ before measuring the turbidity in the CAS medium. As myxobacteria grow slowly in nutrient-rich mediums, whereas other miscellaneous bacteria grow relatively fast, the clear CAS liquid culture medium indicates that the myxobacteria strain is pure. The purified myxobacteria strains were stored in $20 \%$ sterilized glycerol at $-80^{\circ} \mathrm{C}$.

\section{Antagonistic activity of isolated myxobacteria against $P$. infestans}

The plate confrontation method (Li et al. 2011) was used to detect the antagonistic activity of the strains against $P$. infestans. The $P$. infestans that had been cultivated for 7 days was firstly cultured in rye medium for 3 days. Then, the agar block of the pure myxobacteria after 8 days of culture related to a sterile toothpick to a distance of about 2 $\mathrm{cm}$ from the cake of $P$. infestans, and the agar block of sterile VY/2 solid medium was inoculated in the same rye medium as the blank control. The Petri dishes were sealed with a parafilm, and after incubation at $18^{\circ} \mathrm{C}$ for 7 days, the inhibitory zones were observed.

\section{Identification of myxobacteria}

Morphological observation: Referring to Bergey's manual of systematic bacteriology (Boone and Castenholz 2004), the colony morphologies and fruiting bodies of myxobacteria on the $\mathrm{VY} / 2$ medium were observed and photographed.

Molecular identification: The genomic DNA of strain B25-I-1 was isolated using a kit (Rapid Bacterial Genomic DNA Isolation Kit) from Sangon Biotech (Shanghai) Co., Ltd., China, following the instruction provided by the manufacturer. The set of primers (forward 27F: 5'AGAGTTTGATCCTGGCTCAG-3' and reverse 1495R: 5'CTACGGCTACCTTGTTACGA-3') was designed to amplify the partial $16 \mathrm{~S}$ rDNA of myxobacteria (Zhang et al. 2010). The reaction was initiated with denaturation at $94^{\circ} \mathrm{C}$ for $30 \mathrm{~s}$, followed by primer annealing at $55^{\circ} \mathrm{C}$ for $45 \mathrm{~s}$ and primer extension at $72^{\circ} \mathrm{C}$ for $90 \mathrm{~s}$. After 30 cycles of these parameters, an extension step was performed at $72^{\circ} \mathrm{C}$ for 5 min. The PCR products were sent to Beijing Liuhe Huada Gene Technology Co. Ltd. for sequencing, and the $16 \mathrm{~S}$ 
rDNA sequences of the tested strains were compared with known sequences in GenBank using the BLAST software.

\section{Antimicrobial activity of strain B25-I-1}

M. xanthus B25-I-1 was transferred to fresh VY/2 solid medium and incubated at $30^{\circ} \mathrm{C}$ for 7 days. The microorganisms S. aureus, E. coli and B. subtilis were individually added intosterilized beef extract peptone liquid medium and shaken overnight. The next day, after centrifugation, the bacterial pellets were evenly distributed on a beef extract peptone solid medium and allowed to dry. $S$. cerevisiae was grown in YPD liquid culture for $18 \mathrm{~h}$ and then uniformly plated on a potato medium. M. xanthus B25I-1 on the VY/2 solid medium was assembled into microcapsules and inverted onto the beef extract peptone solid medium and potato medium coated with uniform indicator bacteria (Wu et al. 2018). The same amount of the VY/2 medium as a blank control was placed on the beef extracts peptone solid medium and potato medium. After incubation for $48 \mathrm{~h}$ at $37^{\circ} \mathrm{C}$, the antibacterial activities were evaluated.

Using the plate confrontation culture method (Li et al. 2011), F. oxysporum, R. solani, S. sclerotiorum and V. dahliae were individually inoculated on the fresh PDA solid medium. Subsequently, M. xanthus B25-I-1 was assembled into microcapsules and inverted approximately $2.5 \mathrm{~cm}$ from each indicator strain, and the VY/2 solid medium as a blank control was inoculated in the same manner. The Petri dishes were sealed with a parafilm, and after incubation at $18^{\circ} \mathrm{C}$ for 7 days, the inhibitory zones were observed.

\section{Growth curve of strain B25-I-1}

M. xanthus B25-I-1 on the solid medium was transferred into $100 \mathrm{~mL}$ of the VY/2 liquid medium. After shock culture for 3 days at $30^{\circ} \mathrm{C}$ and $180 \mathrm{r} \mathrm{min}^{-1}$, a sterile rotor with magnetic suspension stirring was used to break the bacterial blocks. The cells of $M$. xanthus B25-I-1 were then transferred to $100 \mathrm{~mL}$ of the VY/2 liquid medium at $10 \%$ of the inoculum size, and the culture was conducted by shock culture at $30^{\circ} \mathrm{C}$ and $180 \mathrm{r} \mathrm{min}^{-1}$. Cells were collected every $24 \mathrm{~h}$ and centrifuged at $8000 \mathrm{r} \mathrm{min}^{-1}$ for $10 \mathrm{~min}$. The supernatant was discarded and the bacterial pellet was washed with deionized water and centrifuged again at 8000 $\mathrm{r} \min ^{-1}$ for $10 \mathrm{~min}$. The supernatant was discarded again, and the bacterial pellet was dried in an oven at $50^{\circ} \mathrm{C}$ until the weight was constant. Dry weight of the bacteria was measured three times in parallel. The growth curve of $M$. xanthus B25-I-1 was drawn according to the dry weights (Tang et al. 2014).

\section{Localization of bioactive secondary metabolites from strain B25-I-1}

Similar to the above process, $M$. xanthus B25-I-1 on the solid medium was transferred into $100 \mathrm{~mL}$ of the VY/2 liquid medium. After shock culture for 3 days at $30^{\circ} \mathrm{C}$ and180 $\mathrm{r} \mathrm{min}^{-1}$, a sterile rotor with magnetic suspension stirring were used to break the bacterial blocks. The cells were then transferred into $100 \mathrm{~mL}$ of the VY/2 liquid medium at $10 \%$ of the inoculum size, and culture was conducted by shock culture at $30^{\circ} \mathrm{C}$ and $180 \mathrm{r} \mathrm{min}^{-1}$ for 7 days. The fermentation broth was centrifuged at $8000 \mathrm{r} \mathrm{min}^{-1}$ for $20 \mathrm{~min}$ to separate the supernatant from the bacterial pellet, and the supernatant was collected. The bacterial pellet was washed three times with sterile water and then disrupted in an ultrasonic cell pulverizer isolation box. The lysed cells were centrifuged again, and the supernatant was collected. The extracellular and intracellular supernatants were lyophilized and weighed, dissolved in sterile water to maintain the same concentration of the extracellular and intracellular substances, filtered through a filtration membrane, and evaluated for the resistance activity against $P$. infestans using the filter paper method.

\section{Optimization of the fermentation conditions of strain B25-I-1}

The cultivation conditions, including inoculum size, fermentation period, culture temperature, and rotating speed of the shaking bed, were optimized through a combination of univariate analysis and orthogonal optimization to provide basic data for future industrial production. Table 1 summarizes the single-factor screening scheme for optimizing the culture conditions.

According to the results of the single-factor experiments, the factors and range of the antagonistic activities of $M$. xanthus B25-I-1 against $P$. infestans were determined, and M. xanthus B25-I-1 was then inserted into a seed culture medium at $30^{\circ} \mathrm{C}$ with $180 \mathrm{r}$ min ${ }^{1}$ shaking for 3 days. The inoculum size was set to 8,10 or $12 \%$, the fermentation temperature was set to 28,30 or $32^{\circ} \mathrm{C}$, and the fermentation cycle was set to 7,8 , or 9 days. The L9 $\left(3^{3}\right)$ orthogonal design was used to perform the 3-factor and 3-level orthogonal optimization experiments at $180 \mathrm{r} \min ^{-1}$ shaking. The filter paper method was used to determine the antibacterial activity of the fermentation supernatants.

\section{Stability of the bioactive secondary metabolites}

After fermentation of M. Xanthus B25-I-1 under its optimal conditions, the fermentation broth was centrifuged at $8000 \mathrm{r}$ $\min ^{-1}$ for $20 \mathrm{~min}$ to separate the supernatant and the bacterial pellet, and the supernatant was collected and lyophilized. The lyophilizate was dissolved in sterile water and filtered through a $0.22-\mu \mathrm{m}$ filter, and the fermentation broth was concentrated 50 times. The filter paper method was used to detect the stability of the concentrated fermentation supernatant against temperature, proteinase $\mathrm{K}, \mathrm{UV}$, and natural light, and to determine its capacity for long-term storage. 


\section{Concentration and antimicrobial activities of the} bioactive secondary metabolites

The XAD-16 macroporous adsorption resin of $4 \%$ was added to the VY/2 liquid fermentation medium, followed by the addition of $M$. xanthus B25-I-1. The resin was collected after fermentation under optimal conditions, and 10 volumes of methanol was added after air-drying under natural conditions. The mixture was eluted three times, and the eluate was combined and concentrated under reduced pressure in a rotary evaporator. After evaporation, the sample was weighed, dissolved in a small amount of methanol, filtered through a $0.22-\mu \mathrm{m}$ microporous membrane (organic phase), and placed in a refrigerator at $4^{\circ} \mathrm{C}$. The antagonistic activities of the bioactive secondary metabolites from $M$. xanthus B25-I-1against S. aureus, E. coli, B. subtilis, $S$. cerevisiae, $F$. oxysporum, $R$. solani, and $S$. sclerotiorum were evaluated using the filter paper method.

\section{Antidisease effects of the bioactive secondary metabolites on potato late blight}

Four varieties of potatoes (Qingshu No.9, Jizhangshu, Feurita, and Kexin No.1) were used to detect the antidisease effects of the bioactive secondary metabolites. Qingshu No. 9, Jizhangshu, Feurita, and Kexin No. 1 were grown for 5-8 weeks under light conditions. Healthy and similarly sized leaves of mature plants were cut, rinsed with tap water, and placed in sterile $150-\mathrm{mm}$ dishes, the bottoms of which were moisturized with wet sterile cotton wool. The treatment groups had concentrated samples of 200, $100,10,5,3$, and $0 \mu \mathrm{g} \mathrm{mL}{ }^{-1}$ applied to the back of the leaves. After air-drying, $100 \mu \mathrm{L}$ of the $P$. infestans spore suspension was sprayed onto each leaf. The culture dishes were then placed in a $20^{\circ} \mathrm{C}$ light incubator and cultured for 7-10 days under $16 \mathrm{~h}$ light/ $8 \mathrm{~h}$ dark conditions to observe pathogen infection and measure the diseased areas of the leaves. The zoospore suspensions were replaced by sterile distilled water for the control group 1, the methanol concentrate of the bioactive secondary metabolites was replaced with sterile distilled water for the control group 2 , and six leaves were used for each treatment. The experiment was performed in triplicate.

\section{Results}

Isolation of myxobacteria with antagonistic activity against $P$. infestans

Three strains with the characteristics of myxobacteria were purified from soil samples. All of them showed antagonistic activity against $P$. infestans, and the strain with relatively strong antagonistic activity was named as B25-I-1. The diameter of inhibition zone was about $24 \mathrm{~mm}$. Its activity against $P$. infestans is shown in Fig. 1.
Table 1: Screening program of single factors for fermentation conditions

\begin{tabular}{llllll}
\hline Factors & \multicolumn{5}{c}{ Levels } \\
\hline Incubation temperature ${ }^{\circ} \mathrm{C}$ & 25 & 28 & 30 & 32 & 35 \\
Incubation time d & 5 & 6 & 7 & 8 & 9 \\
Inoculum size $\%$ & 5 & 8 & 10 & 12 & 15 \\
Rotating speed r min $^{-1}$ & 150 & 160 & 170 & 180 & 190 \\
\hline
\end{tabular}

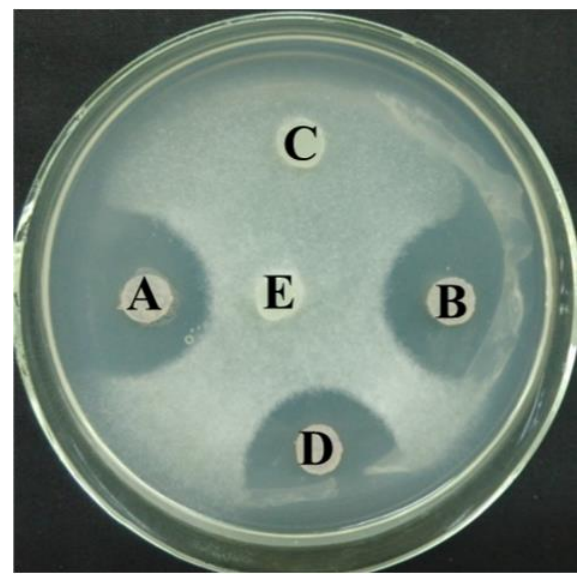

Fig. 1: The antibiotic activity of the strain B25-I-1 against $P$. infestans

(A/B/D) The strain B25-I-1 (C) Blank control (E): P. infestans

\section{Identification of the strain B25-I-1}

Morphological identification: Strain B25-I-1could form characteristic colonies on the VY/2 medium, with a thin and translucent membrane that was circularly expanded. The fruiting bodies were soft, single-growth, irregularly distributed, yellow, ellipsoidal or spherical and did not have a stalk-like structure but had refraction and transparent mucus on their periphery (Fig. 2). The morphological features of the strain B25-I-1 were similar to those of Myxococcus xanthus according to Bergey's manual of systematic bacteriology.

Molecular identification: The sequence analysis of the $16 \mathrm{~S}$ rRNA gene fragment of the strain B25-I-1 was performed, and a phylogenetic tree was constructed by the neighborjoining method (Fig. 3). The phylogenetic tree indicated that the strain B25-I-1 was Myxococcus; it had the highest similarity to the $M$. xanthus A19 (accession number DQ411303), and they clustered in the same phylogenetic evolution branch. Taken together with these morphological characteristics, the strain B25-I-1 was identified as $M$. xanthus (accession number MH730556).

\section{Antimicrobial spectrum of $M$. xanthus B25-I-1}

M. xanthus B25-I-1 can kill and dissolve E. coli and can inhibit the growth of B. subtilis, F. oxysporum, $R$. solani, $S$. sclerotiorum, $V$. dahliae, and $P$. infestans. It had the strongest inhibitory activity against $P$. infestans but did not show activity against $S$. aureus or $S$. cerevisiae (Table 2). 

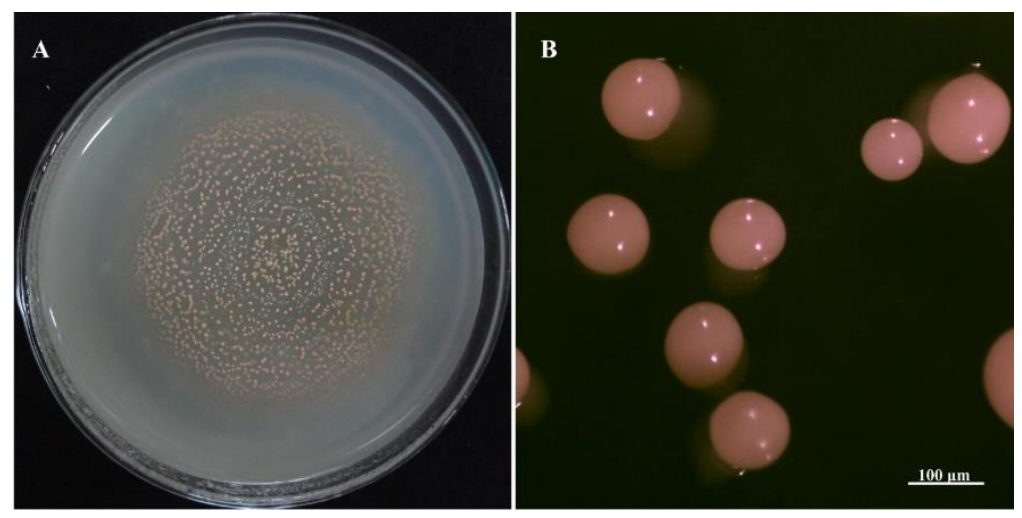

Fig. 2: The morphological characteristics of the strain B25-I-1

(A) Colony (B) Fruiting bodies

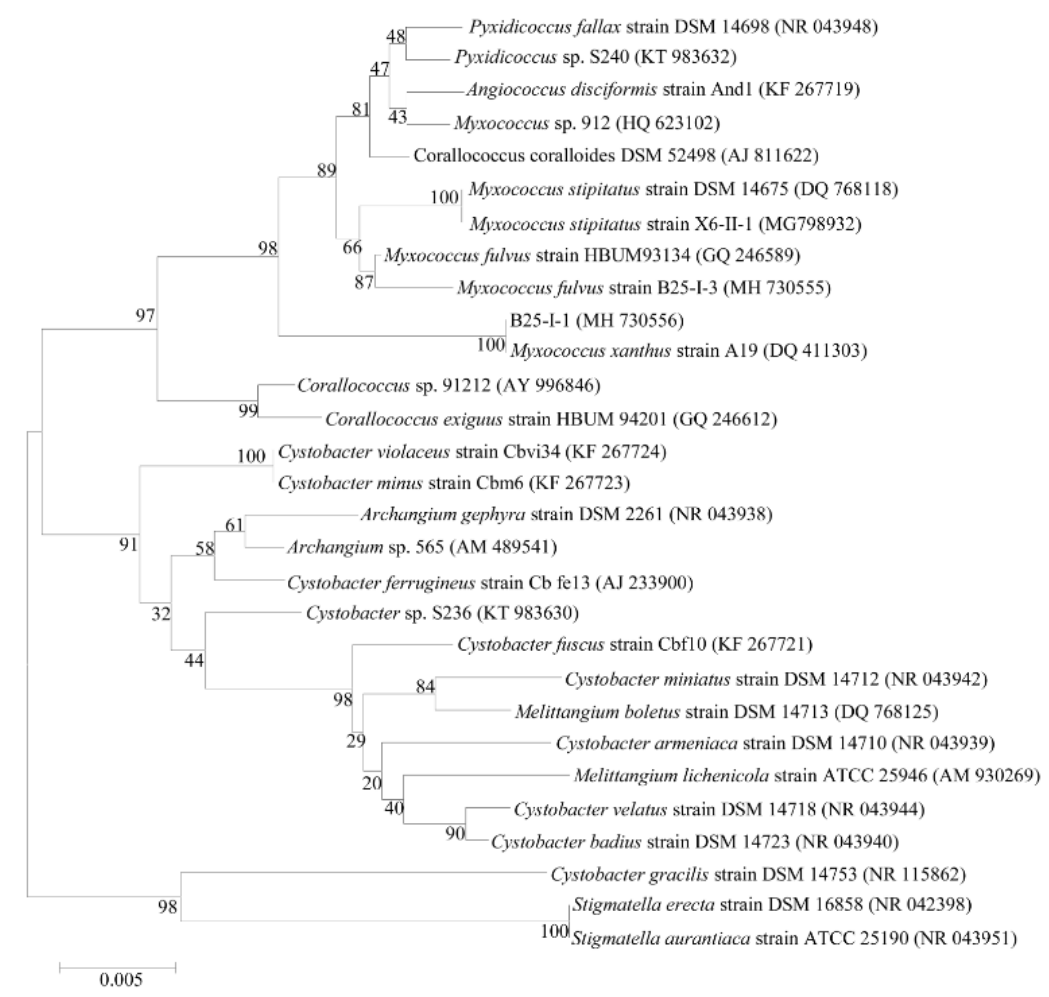

Fig. 3: The phylogenetic tree of the strain B25-I-1 based on the 16S rRNA gene sequence

\section{Determination of growth curve and distribution of secondary metabolites}

As shown in Fig. 4A, the growth of M. xanthus B25-I-1 was delayed for 3 days after inoculation, after which the weight of the bacteria began to increase. On the $6^{\text {th }}$ day after inoculation, the weight of the cells reached a maximum, then reduced rapidly and stabilized after 7 days. After $M$. xanthus B25-I-1 was fermented and cultured, the filter paper method was used to detect the resistance to $P$. infestans induced by the intracellular and extracellular substances. As shown in Fig. 4B, the extracellular substances of $M$. xanthus B25-I-1 showed an inhibitory effect on the growth of $P$. infestans; however, antagonistic activity of the intracellular material was not detected, thus suggesting that the bioactive secondary metabolites of $M$. xanthus B25- I-1 against $P$. infestans exist outside the cell.

\section{Optimization of the fermentation conditions}

Effects of inoculum size, as shown in Fig. 5A, with an increase in the inoculum size, the antagonistic activity of the fermentation supernatant on $P$. infestans increased initially before decreasing. The antagonistic activity was highest when the inoculum size was $10 \%$, and the diameter of inhibition zone was $12.5 \mathrm{~mm}$. Therefore, in this experiment, 
Table 2: The antimicrobial spectrum of M. xanthus B25-I-1

\begin{tabular}{lc}
\hline Indicator microorganism & Antibiotic activity \\
\hline Escherichia coli & ++ \\
Bacillus subtilis & + \\
Staphylococcus aureus & - \\
Saccharomyces cerevisiae & - \\
Fusarium oxysporum & + \\
Rhizoctonia solani & ++ \\
Sclerotinia sclerotiorum & ++ \\
Phytophthora infestans & +++ \\
Verticillium dahliae & + \\
\hline
\end{tabular}

(+++) strong inhibition; (++) medium inhibition; (+) weak inhibition; (-) no inhibition
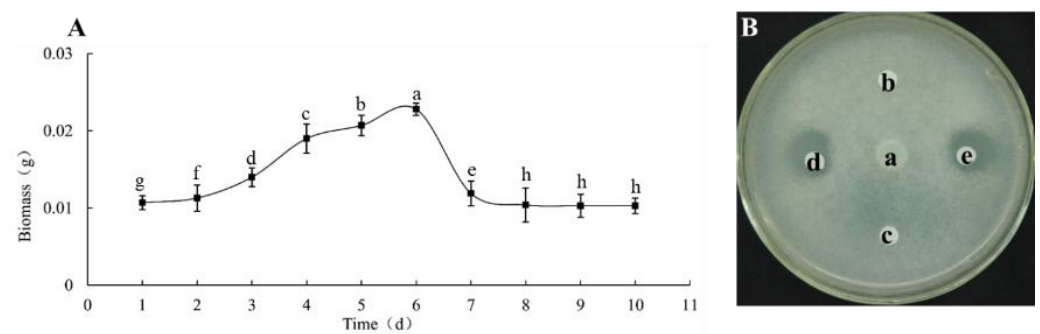

Fig. 4: The growth curve and distribution of the bioactive secondary metabolites of $M$. xanthus B25-I-1

(A) The growth curve (B) distribution of bioactive secondary metabolites (a) P. infestans (b) blank control (c) intracellular substances (d/e) extracellular substances. The lowercase letters indicate significant difference level of $\mathrm{p} 0.05$
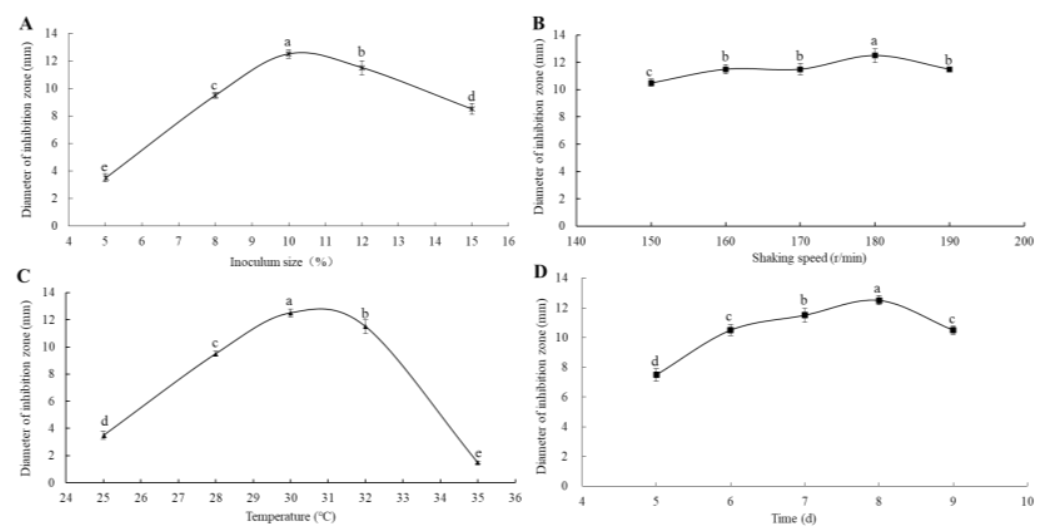

Fig. 5: Effects of the inoculum size (A), shaking speed (B), incubation temperature (C), and incubation time (D) on the antibiotic activity of the fermentation supernatant

The lowercase letters indicate significant difference level of $\mathrm{p} 0.05$

the inoculum size of $10 \%$ was selected as the optimum inoculum size.

Effects of the shaker speed: During the fermentation of M. xanthus B25-I-1, the effects of different rotation speeds on the antagonistic activity of the fermentation supernatant were minimal. As shown in Fig. 5B, the antagonistic activity generally increased over the range of $150-180 \mathrm{r} \mathrm{min}^{-1}$, reaching the highest antagonistic activity against $P$. infestans at $180 \mathrm{r} \mathrm{min}^{-1}$. At $180-190 \mathrm{r} \mathrm{min}^{-1}$, the antagonistic activity was stable; therefore, the optimum speed was determined to be $180 \mathrm{r} \mathrm{min}^{-1}$.

Effects of fermentation temperature, as shown in Fig. $5 \mathrm{C}$, the antagonistic activity of the fermentation supernatant increased over a temperature range of $25-30^{\circ} \mathrm{C}$. The antagonistic activity reached maximum at $30^{\circ} \mathrm{C}$ when the diameter of inhibition zone was $12.5 \mathrm{~mm}$. The antagonistic activity then decreased with a further increase in the temperature, indicating that higher or lower temperatures were not conducive to antagonistic activity. Therefore, $30^{\circ} \mathrm{C}$ was selected as the optimal fermentation temperature for $M$. xanthus B25-I-1.

Effects of fermentation time, as shown in Fig. 5D, the antagonistic activity of $M$. xanthus B25-I-1 was low after 5 days of fermentation, but the antagonistic activity increased with prolonged fermentation time. The antagonistic activity was maximum on the $8^{\text {th }}$ day, and it began to decline by the $9^{\text {th }}$ day. Ultimately, the optimal fermentation time was determined to be 8 days. 
Optimization of Anti P. infestans Metabolites / Intl J Agric Biol, Vol 25, No 6, 2021

Table 3: Orthogonal experiment design and results for M. xanthus B25-I-1

\begin{tabular}{|c|c|c|c|c|}
\hline \multirow[t]{2}{*}{ Test number } & \multicolumn{3}{|c|}{ Factors } & \multirow{2}{*}{$\begin{array}{l}\text { Diameter of inhibition zone }(\mathrm{mm}) \\
\text { Average value }\end{array}$} \\
\hline & Temperature $\left({ }^{\circ} \mathrm{C}\right)$ & Incubation time (d) & Inoculum size (\%) & \\
\hline 1 & 28 & 8 & 12 & $10.5 \mathrm{~d}$ \\
\hline 2 & 28 & 9 & 8 & $10.9 \mathrm{~d}$ \\
\hline 3 & 30 & 7 & 12 & $10.5 \mathrm{~d}$ \\
\hline 4 & 30 & 9 & 10 & $11.9 \mathrm{c}$ \\
\hline 5 & 30 & 8 & 8 & $12.1 \mathrm{c}$ \\
\hline 6 & 32 & 9 & 12 & $13.5 \mathrm{~b}$ \\
\hline 7 & 32 & 7 & 8 & $13.9 \mathrm{~b}$ \\
\hline 8 & 28 & 7 & 10 & $9.5 \mathrm{e}$ \\
\hline 9 & 32 & 8 & 10 & $15.1 \mathrm{a}$ \\
\hline Kl & 10.3 & 11.3 & 12.3 & $K=12$ \\
\hline$K 2$ & 11.5 & 12.6 & 12.2 & \\
\hline K3 & 14.2 & 12.1 & 11.5 & \\
\hline$R$ & 3.9 & 1.3 & 0.8 & \\
\hline
\end{tabular}

Table 4: Variance analysis of orthogonal optimization results for M. xanthus B25-I-1

\begin{tabular}{|c|c|c|c|c|c|}
\hline Source of variance & Square sum of the mean squared & Degrees of freedom & Mean square & $\mathrm{F}$ & $P$ \\
\hline Temperature & 0.976 & 2 & 0.488 & 15.679 & 0.060 \\
\hline Incubation time & 0.762 & 2 & 0.381 & 12.250 & 0.075 \\
\hline Inoculum size & 0.996 & 2 & 0.498 & 16.000 & 0.059 \\
\hline Deviation & 0.062 & 2 & 0.031 & & \\
\hline
\end{tabular}

On the basis of the above single-factor experiment results, three factors that had a great influence on the antagonistic activity of the fermentation supernatant of $M$. xanthus B25-I-1 were selected. The inoculum size, fermentation temperature, and fermentation time were orthogonalized. The three factors were selected at three levels for the experimental design. The program and results are shown in Table 3. The fermentation supernatant produced at different temperatures, times, and inoculum sizes had different levels of antagonism to $P$. infestans. The optimum fermentation conditions to produce bioactive secondary metabolites against $P$. infestans were as follows: temperature of $32^{\circ} \mathrm{C}$, time of 8 days, and inoculum size of $10 \%$. From the size range analysis, the order of the ability of the supernatant of $M$. xanthus B25-I-1 to antagonize $P$. infestans was temperature $>$ time $>$ inoculum size.

The results using the range calculation cannot be used to quantitatively analyze the error. It was not possible to determine whether the differences between each level of each factor were caused by experimental error or whether there was a substantial difference between the levels of each factor. Therefore, to further reflect the differences among the factors used to optimize the optimal fermentation conditions for $M$. xanthus B25-I-1 to produce substances antagonistic to $P$. infestans, analysis of variance was used to analyze the results, as shown in Table 4. Among the variables, the $P$ value for temperature was $<0.05$ and close to 0.01 , which represented a significant effect on the experimental results. Therefore, temperature was considered the primary factor, and fermentation time and inoculum size were considered secondary factors. Variance analysis was then used to compare the $\mathrm{F}$ values, and it was further determined that temperature was the main factor influencing the activity of the fermentation products, and the effects of the fermentation time was slightly greater than those of the inoculum size. Therefore, the effects of the culture temperature, fermentation time, and inoculum size on the activity against $P$. infestans was ordered as temperature > time > inoculation amount, which is consistent with the range analysis results.

\section{Stability tests of the bioactive secondary metabolites}

Thermal stability: The bioactive secondary metabolites against $P$. infestans maintained high antagonistic activity after being treated for $30 \mathrm{~min}$ at temperatures of $30-100^{\circ} \mathrm{C}$ (Fig. 6). Compared with the antagonistic activity of the bioactive secondary metabolites stored at $4^{\circ} \mathrm{C}$, the activities against $P$. infestans were slightly reduced after treatment at $30-50^{\circ} \mathrm{C}$ for $30 \mathrm{~min}$. However, after treatment for $30 \mathrm{~min}$ at $60-100^{\circ} \mathrm{C}$, the activities had an increasing tendency. We speculated that the solvent was mainly evaporated due to the higher temperature to increase the concentrations of the bioactive secondary metabolites. It can be preliminarily concluded that temperature has little effect on the activity of secondary metabolites to inhibit the growth of $P$. infestans.

Proteinase K, UV light, natural light, and storage temperature stability: The concentrated fermentation broth of M. xanthus B25-I-1 was treated with proteinase $\mathrm{K}$ for $1 \mathrm{~h}$, $\mathrm{UV}$ light for $1 \mathrm{~h}$, and natural light for 1 hand then compared with the concentrated fermentation broth. There was no significant change in the size of the inhibition zone for the growth of $P$. infestans (Fig. 7A). It can be preliminarily indicated that the secondary metabolites were stable to UV light, proteinase $\mathrm{K}$, and natural light, which also suggests that the bioactive secondary metabolites of M. xanthus B25- 
Table 5: Antibiotic spectrum of the bioactive secondary metabolites of M. xanthus B25-I-1

\begin{tabular}{lc}
\hline Indicator microorganism & Antibiotic activity \\
\hline Escherichiacoli & + \\
Bacillus subtilis & + \\
Staphylococcus aureus & + \\
Saccharomyces cerevisiae & + \\
Fusarium oxysporum & + \\
Rhizoctonia solani & + \\
Sclerotinia sclerotiorum &
\end{tabular}

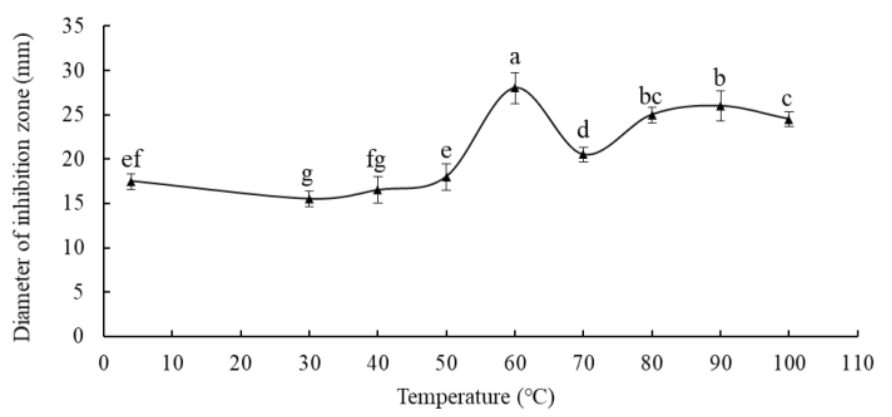

Fig. 6: Effect of temperature on the antibiotic activity of the metabolites of M. xanthus B25-I-1 The lowercase letters indicate significant difference level of $\mathrm{p} 0.05$
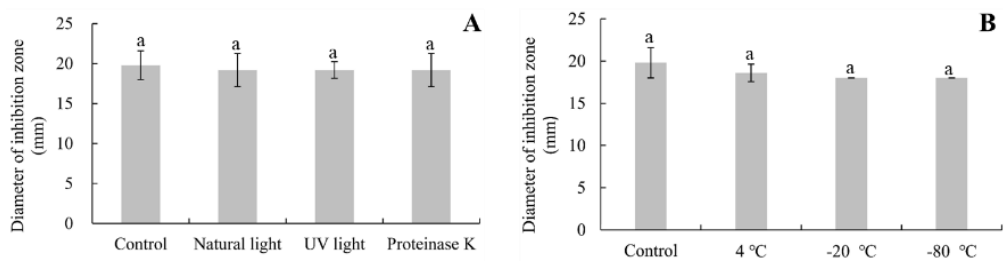

Fig. 7: Effects of proteinase K, UV light, natural light, and storage temperature on the antibiotic activity of the metabolites. The lowercase letters indicate significant difference level of 0.05

I-1against $P$. infestans is not protein based. The activity of the bioactive secondary metabolites against $P$. infestans decreased slightly after storage for 15 days at-80, -20 and $4^{\circ} \mathrm{C}$, but the decrease was not significant (Fig. 7B). The results indicated that the secondary metabolites produced by M. xanthus B25-I-1 facilitated long-term storage at -80, -20 and $4^{\circ} \mathrm{C}$.

\section{Antimicrobial spectrum of the bioactive secondary metabolites}

The secondary metabolites produced by $M$. xanthus B25-I-1 that inhibited the growth of $P$. infestans demonstrated inhibition of the bacteria $E$. coli, S. aureus, and B. subtilis and fungi $S$. cerevisiae, $F$. oxysporum, $R$. solani, and $S$. sclerotiorum (Table 5). However, M. xanthus B25-I-1 showed no activity against $S$. aureusor $S$. cerevisiae. We speculate that the bioactive secondary metabolites secreted by $M$. xanthus B25-I-1 were at low levels, but after the fermentation liquid was concentrated, the concentration of the bioactive secondary metabolites increased, thus showing a broader spectrum of antimicrobial activity than the unconcentrated material.

\section{Antidisease activity of the bioactive secondary metabolites on potato late blight}

The relative lesion areas of different varieties of potato leaves were measured using the Adobe Photoshop CS5 software (Cui et al. 2009), and the results are shown in Fig. 8. In the control groups treated with only sterile water and only methanol, the percentage of damaged areas of the leaves of the four different varieties of potatoes was over $90 \%$ after infection with $P$. infestans. In the test groups treated with different concentrations of the bioactive secondary metabolites from $M$. xanthus B25-I-1, the relative lesion areas of the leaves of the four varieties of potatoes decreased gradually with increasing of concentrations of the bioactive secondary metabolites, indicating that the bioactive secondary metabolites had obvious disease prevention effects on the potato leaves in vitro, and the disease prevention effects were not specific to the variety of the potatoes.

\section{Discussion}

The use of beneficial microorganisms to restrict the harmful microorganisms is an important area of research in the 

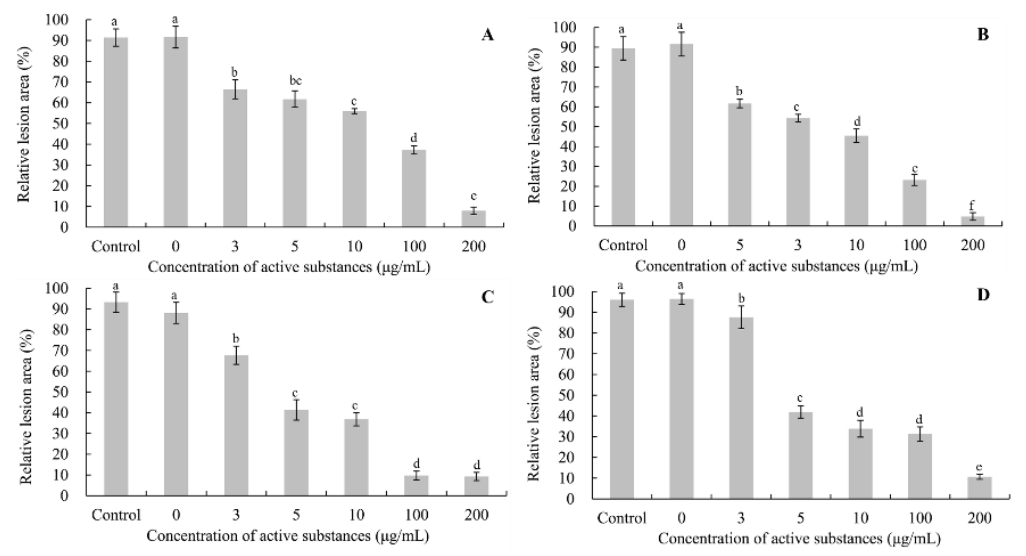

Fig. 8: Effects of the bioactive secondary metabolites on the relative lesion areas of the detached potato leaves (A) Jizhangshu (B) Feurita (C) Kexin No.1 (D) Qingshu No.9The lowercase letters indicate significant difference level of p 0.05

development and utilization of microbial resources and is one of the most promising methods to control plant diseases (Jiang et al. 2001). In this work, the M. xanthus strain B25-I-1 was successfully isolated from a soil sample and showed strong antagonistic activity against $P$. infestans. Abbas et al. (2009) isolated a variety of secondary metabolites from Trichoderma that significantly inhibited plant pathogens. Caulier et al. (2018) found that B. subtilis 30B-B6 can also produce resistance to $P$. infestans and can significantly reduce the occurrence of plant diseases. Jiang et al. (2010) found that the composite fermentation broth of three strains of actinomycetes against $P$. infestans significantly inhibited the mycelial growth of pathogenic bacteria and caused mycelial deformation or abnormality of sporangia. These studies have shown that there is a great potential to develop a biopesticide against potato late blight from biologically active secondary metabolites produced by microorganisms.

The present study focused on myxobacteria, which are known as the "microfactories" of bioactive secondary metabolites. At present, approximately 600 biologically active substances have been identified from myxobacteria, which have antibacterial, antiviral, anticancer, and thrombolytic activities. These substances represent many novel structures, levels of action, and unique mechanisms of action. For example, chivosazol (Irschik et al. 1995) can destroy the integrity and stability of fungal cell walls. Myxochelin (Silakowski et al. 2000) interferes with the transport of metal ions from bacteria and some fungi. Disorazol (Khalil et al. 2006) acts on tubulin to inhibit cytoskeletal formation. Myxopyronin (Audrey et al. 2009), inhibits nucleic acid synthesis primarily by inhibiting bacterial RNA polymerase. These results illustrate the possibility of using myxobacteria to inhibit the growth of $P$. infestans. The secondary metabolites of $M$. xanthus have attracted increasing attention, but little has been reported about the use of its secondary metabolites in the prevention and control of plant diseases, especially potato late blight. In the present study, the M. xanthus B25-I-1 showed a strong antagonistic activity against $P$. infestans, and the bioactive secondary metabolites produced by its fermentation significantly inhibited the infection of detached potato leaves caused by $P$. infestans and caused no damage to the leaves of different potato varieties by themselves. These results complement the existing microbial resources that inhibit the growth of $P$. infestans and provide basic data for the future development of biopesticides against potato late blight.

Although the bioactive secondary metabolites produced by myxobacteria are quite varied (various types, wide resistance, novel structure, etc.), factors such as the secretion of mucus and autolysis by extracellular enzymes limit the fermentation and production of bioactive secondary metabolites by these organisms. The optimization of the fermentation conditions mainly includes factors such as fermentation temperature, time, rotation speed, feeding, and ventilation, and these factors generally do not affect the fermentation of the strain independently but interact with each other (Zhang 2011). Xie et al. (2017) applied an orthogonal design to optimize the fermentation parameters of Lactobacillus plantarum r13 and increased the biomass and effective viable concentration of the bacteria solution. Feng et al. (2016) systematically optimized the fermentation and culture conditions of $B$. thuringiensis PanD37 by orthogonal tests, and the L-aspartate $\alpha$-deacidase activity of the strain was greatly improved. These findings indicate that the biological activities of microorganisms can be improved by optimizing the fermentation conditions. In the present study, the fermentation conditions of $M$. xanthus B25-I-1 were orthogonally optimized for activity against $P$. infestans. The optimal fermentation conditions were as follows: inoculum size of $10 \%$, shaker speed of $180 \mathrm{r} \mathrm{min}^{-1}$, culture temperature of $32^{\circ} \mathrm{C}$, and fermentation time of $8 \mathrm{~d}$. Under the above optimized culture conditions, the antibacterial activity of the fermentation supernatant was determined using the filter paper method and the growth edge of the $P$. infestans was found to be up to $7.3 \mathrm{~mm}$ from the filter paper. This work lays the foundation for future developments of biopesticides against potato late blight 
based on the M. xanthus B25-I-1.

In addition, the present study found that the bioactive secondary metabolites were highly tolerant to high temperature, treatment with protease $\mathrm{K}$, and UV and natural lights and that these substances could be preserved for long periods at a low temperature. These results were consistent with the results for the $M$. virescens YR-35 fermentation broth observed by Ren et al. (2016). However, inhibition of $P$. infestans caused by the bioactive secondary metabolites of YR-35 reached only $15.38 \%$ after incubation at $90^{\circ} \mathrm{C}$, while a high temperature had little effect on the bioactive secondary metabolites of $M$. Xanthus B25-I-1. The secondary metabolites of $M$. fulvus xt-2 with antipathogenic $P$. infestans activity isolated by Liu et al. (2014) were also sensitive to high temperature. Conversely, the active products of M. xanthus B25-I-1 have the potential to control potato late blight at ahigh temperature found in the fields.

The findings of the present study demonstrated that the secondary metabolites of the M. xanthus B25-I-1 have practical value and can be developed and utilized as an effective agent for controlling potato late blight. However, the type of bioactive secondary metabolites, their mechanisms for antagonizing $P$. infestans, and their application in the field still need to be studied further.

\section{Conclusion}

In this study, three strains of myxobacteria were isolated from the soil sample collected from Baotou area of Inner Mongolia Autonomous Region. Among them, the strain B25-I-1 showed strong antagonistic activity against $P$. infestans and was identified as M. xanthus. This strain could kill and dissolve E. coli, and showed a different degree of inhibition to $B$. subtilis, $F$. oxysporum, $R$. solani, $S$. sclerotiorum and $V$. dahlia. The optimal fermentation conditions for the active substances produced were as follows: inoculum size $10 \%$, shaking speed $180 \mathrm{r} \mathrm{min}^{-1}$, incubation temperature $30^{\circ} \mathrm{C}$, incubation time 8 days. The active substances were highly tolerant to temperature, the treatment of protease $\mathrm{K}$, ultraviolet light and natural light. The active substances exhibited different degrees of antagonism against $E$. coli, S. aureus, B. subtilis, $S$. cerevisiae, $F$. oxysporum, $R$. solani, and $S$. sclerotiorum. The active substances could inhibit the infection of the detached potato leaves by $P$. infestans significantly and had no damage to the leaves of different varieties of potato. $M$. xanthus $\mathrm{B} 25-\mathrm{I}-1$ can produce the active substances against $P$. infestans, and has the potential value of developing biological pesticide against potato late blight. This study provides basic data for the development of biopesticide against potato late blight.

\section{Acknowledgements}

The authors acknowledge the efforts of Ye Dong and $\mathrm{Zi}$ Wen Guo for isolating the microorganism.

\section{Author Contributions}

Wu ZH and Liu HR planned the experiments, Wu ZH, Zhao $\mathrm{PY}$ and Ding YX interpreted the results, $\mathrm{Wu} \mathrm{ZH}, \mathrm{Ma} \mathrm{Q}$ and Wang $\mathrm{XH}$ made the write up and $\mathrm{Wu} \mathrm{ZH}$ statistically analyzed the data and made illustrations.

\section{Conflict of Interest}

The authors declare that they have no conflicts of interest, and manuscript is approved by all authors for publication. There is no conflict of interest among the institutions regarding the research when it has been conducted at the institutions other than authors institutions. If such a conflicting situation arises, the authors will be held responsible.

\section{Data Availability}

The datasets generated during and/or analysed during the current study are available from the corresponding author on reasonable request.

\section{Ethics Approval}

This article does not contain any studies with human participants or animals performed by any of the authors.

\section{References}

Abbas EH, W Frank, S Jochen, B Heinrich (2009). Detection of viridiofungin $\mathrm{A}$ and other antifungal metabolites excreted by Trichoderma harzianum active against different plant pathogens. Eur J Plant Pathol 124:457-470

Audrey T, G Maxime, B Konstantin, JP Leonetti (2009). Myxopyronin: A punch in the jaws of bacterial RNA polymerase. Future Microbiol 4:145-149

Boone DR, RW Castenholz (2004). Bergey's Manual of Systematic Bacteriology. Springer-Verlag, New York, USA

Caulier S, A Gillis, G Colau, F Licciardi, M Liépin, N Desoignies, P Modrie, A Legreve, J Mahillon, C Bragard (2018). Versatile antagonistic activities of soil-borne Bacillus spp. and Pseudomonas spp. against Phytophthora infestans and other potato pathogens. Front Microbiol 9; Article 143

Cui HW, YL Yang, JT Li, WF Luo, AM Miao, ZX Hu, XN Han (2009). A faster method for measuring relative lesion area on leaves based on software photoshop. J Anhui Agric Sci 37:10760-10762

Diez J, JP Martinez, J Mestres, F Sasse, R Frank, A Meyerhans (2012). Myxobacteria: Natural pharmaceutical factories. Microb Cell Fact 30:11-52

Feng ZB, J Zhang, GZ Chen, YP Cha, JJ Liu, YH Ge, SW Chen, BT Yu (2016). Isolation, identification and fermentation optimization of Bacillus tequilensis PanD37 producing L-aspartate $\alpha$-decarboxylase. Acta Microbiol Sin 56:44-55

Fry W (2008). Phytophthora infestans: The plant (and R gene) destroyer. Mol Plant Pathol 9:385-402

Guo WJ, GJ Tao, WY Tao, FJ Cui, XC Jin, F Bi, ZH Xu, ZH Ao (2007). A myxobacterium strain Sorangium cellulosum AHB125 producing epothilone B and other anticancer substances. Nat Prod Res 21:1256-1265

Haas BJ, S Kamoun, MC Zody (2009). Genome sequence and analysis of the Irish potato famine pathogen Phytophthora infestans. Nature 461:393-398 


\section{Optimization of Anti P. infestans Metabolites / Intl J Agric Biol, Vol 25, No 6, 2021}

Herrmann J, AA Fayad, R Müller (2017). Natural products from myxobacteria: Novel metabolites and bioactivities. Nat Prod Rep $34: 125-160$

Irschik H, R Jansen, K Gerth, G Höfle, H Reichenbach (1995). Chivosazol A, a new inhibitor of eukaryotic organisms isolated from myxobacteria. J Antibiot 48:962-966

Jiang JZ, S Li, HX Wang (2010). Culture condition optimization of three actinomyces mixed fermentation against Phytophthora infestans and mechanism. J Hebei Univ 30:78-82

Jiang JZ, LK Zhao, J Shi, JG Dong (2001). Inhibition of fungal fermented filtrates on Phytophthora infestans. Microbiol Chin 28:55-59

Kim YJ, HJ Kim, GW Kim, K Cho, S Takahashi, H Koshino, WG Kim (2016). Isolation of coralmycins A and B, potent anti-gram negative compounds from the myxobacteria Corallococcus coralloides M23. J Nat Prod 79:2223-2228

Khalil MW, F Sasse, H Lünsdorf, YA Elnakady, H Reichenbach (2006). Mechanism of action of tubulysin, an antimitotic peptide from myxobacteria. Chem Biol Chem 7:678-683

Li LY, JZ Jiang, W Guo (2011). Screening of antagonistic bacteria against Phytophthora infestans and its inhibition effect. Plant Dis Pests 2:49-55

Liu BH, L Yang, LF Feng, LX He (2011). Isolation and purification of myxobacteria in Chengdu. Anim Husb Feed Sci 3:25-27

Liu PS, N Li, FY Feng, HR Liu (2014). Identification of a myxobacterium strain against Phytophthora infestansand its activity analysis. Sci Technol Eng 14:40-45

Livingstone PG, RM Morphew, DE Whitworth (2018). Genome sequencing and pan-genome analysis of 23 Corallococcus spp. strains reveal unexpected diversity, with particular plasticity of predatory gene sets. Front Microbiol 9; Article 3187

Ren XB, ZL Zhang, PY Zhao, ZH Wu, HC Cui, HR Liu (2016). Isolation and identification of the strain YR-35 resistant to Phytophthora infestans and its metabolites. Chin J Biol Cont 32:379-387
Silakowski B, B Kunze, G Nordsiek, H Blöcker, G Höfle, R Müller (2000). The myxochelin iron transport regulon of the myxobacterium Stigmatella aurantiaca Sg a15. Eur J Biochem 267:6476-6485

Sun YW, T Tomura, J Sato, T Iizuka, R Fudou, M Ojika (2016). Isolation and biosynthetic analysis of haliamide, a new PKS-NRPS hybrid metabolite from the marine myxobacterium Haliangium ochraceum. Molecules 21:59-66

Tang SJ, R Xiao, Y Wen, H Qin, W Liu, XZ Ding, LQ Xia (2014). Isolation and identification of Myxobacteria strain STXZ54 with antitumor activities. Acta Microbiol Sin 54:532-542

Wang DT, ZL Mang (2010). The molecular biology of myxobacterium. Chem Life 30:779-782

Wenzel SC, R Müller (2009). Myxobacteria-'microbial factories' for the production of bioactive secondary metabolites. Mol Biosyst 5:567574

Weissman KJ, R Müller (2009). A brief tour of myxobacterial secondary metabolism. Bioorg Med Chem 17:2121-2136

Wu ZH (2018). Isolation of Myxobacteria from the central region of inner mongolia and their activity and components against potato late blight pathogen. Dissertation. Inner Mongolia Agricultural University, China

Wu ZH, PY Zhao, YX Ding, Q Ma, XH Wang, HR Liu (2020). Identification and secondary metabolites of strain B25-I-3 against Phytophthora infestans. Microbiol Chin 47:3586-3599

Wu ZH, N Li, XZ Ma, Y Dong, ZW Guo, HR Liu (2018). Diversity and bioactivities of myxobacteria in Daxing'an Mountains. Microbiol Chin 45:266-283

Xie FX, FF Zhang, K Zhou, YJ Zhao, Q Zhao, HB Sun (2017). Isolation, identification and fermentation optimization of lactic acid bacteria for aquaculture water purification. Acta Microbiol Sin 57:304-314

Zhang ZD, YQ Xie, M Chu, MY Gu, SQ Song, QY Tang, J Mao (2010). Study on isolation and identification of endophytes in yam rhizome. Xinj Agric Sci 47:126-129

Zhang YT (2011). The studies on isolation, identification and antibacterial activity of Myxococcus fulvus. Dissertation. Henan University of Technology, China 